impaction and moderate deformity, in most fractures of the upper extremity of the humerus, and in the great majority of fractures- of the neck of the femur. In all fractures in which the displacement is due to muscular contraction any attempt at reduction is useless, as in fracture of the clavicle, of the olecranon, and many fractures of the malleoli. After massage the fracture is either spontaneously reduced, as in the case of the olecranon, or is very easy to maintain reduced, as in the case of the clavicle and malleolus. Those conditions which favour the vitality of the limb ara all more important than those which produce immobilization of the fragments. The fact that shortening has not the injurious influence on repair which it is said to have should not be lost sight of when considering dísplacement. A moderate degree of shortening which does not change the static position of a limb is farourable to repair. The muscles surrounding a shortened lever act more rapidly and better than upon a lever on which they are stretched. A certain amount of shortening ought not to be interfered with, as it is favourable to the repair of a broken bone.

Massage has so great an effect in stimulating bony secretion at the site of fracture that in children, in whom the activity of the production of bone is great, massage ought not to be applied, save with great moderation, for fear of provoking excessive osseous secretion-in fact, a tumour. In their case simple mobilization is often sufficient.

Mobilization from the beginning, within the first twentysfour or forty.eight hours, is of capital importance, and: at this stage movements of very small amplitude are sufficient; passive or -induced movements are the best at the beginning. Active or spontaneous movements ought to be measured and directed by the surgeon; to allow the patient to follow his own fancy with regard to active movements is dangerous - all the more dangerous because with my method the pain caused by movement disappears from the beginning, and therefore does not limit the untimely action of voluntary movement. The pain experienced and the degree of sensitiveness of the callus are the best guides in directing and in permitting movement by the patient.

The secondary treatment of fractures and their supervision ought to be prolonged. Functional use of a fractured limb is the condition most favourable to its regaining actioe power. Even during this secondary period everything likely to irritate the region of the callus ought to be avoided. It is useless to cause pain by movements exaggerated in amplitude or by resort to forcible manipulation.

While before my investigations the surgeon contented himself, after having reduced a fracture, by awaiting cure, the modern surgeon should play a much more active, much more personal, and much less simple part. He ought not only to diagnose the fracture but ought to make its exact nature visible by a radiographic picture, and then take action to obtain not only solidity of the limb, but also to restore its suppleness, its regularity of action, and its power. He must not merely await the solidification of the skeleton. Moreover, his treatment must vary a great deal according to

\section{The age,}

The variety in the site of the fracture,

The kind of fracture of the limb,

The general or local health of the patient,

His occupation,

and aocording also to the circumstances under which he is called upon to, act, for if he cannot ensure proper personal supervision of mobilization, massage, and the supervision of the morements, it may happen that he will be compelled to replace an excellent method of mobilization by a moderately good method of suture, or absolute immobilization.

The surgeon needs a great deal of experience to conduct the treatment of a frocture in the best way, and I cannot help asking with some anxiety how a non-medical tribunal is to form a judgement on questions so complex, should the patient and his medical attendants cease to be in accord.

The situation is complicated. Well-to-do people bring actions against surgeons because they continue to experience pain or stiffness after a fracture; workmen reproach the surgeon because he cures them too well, inasmuch as by causing the disappearance of all secondary pain, accidents are not followed by the permanent infirmity for which they hoped.

The whole history of the surgery of fractures cannot be related in an address which is already too long, but I shall have succeeded in my purpose if I have made plain to you to how great an extent the duty of the surgeon has become, difficult indeed, but effective in this matter of fractures, Instead of waiting for the injured person to cure himself, we can cure him and quickly restore to him the use of his limb.

It is a laborious task, more difficult than the banal application of irremovable appliances; it is still insufficiently understood by the profession, and altogether unknown to the public. But I am proud to think that in your country it has met so favourable a reception that you have been willing to listen to the exposition of the subject which I have to-day offered to you as a tribute of fraternal homage.

\section{An Andress \\ oN THE}

\section{PRODROMAS OF MIGRAINE.}

Delivered before the Westminster Division of thB British Medical association.

BY

Sir WILLIAM R. GOWERS, M.D.Lond., F.R.S.

Gentlemen,-I have chosen a subject for your consideration with which every. practitioner is familiar from the accounts of his patients, and a good many are also well acquainted from personal suffering-the curious sensory symptoms that constitute the premonition of attacks of migraine, using the term as equivalent to paroxysmal headache, The symptoms occur before every form of such headaches, those which are bilateral, as well as those that may be strictly termed " hemicrania," but both are common without premonition.

The knowledge we have of these peculiar symptoms, the prodromas of migraine, is very small; compared with their frequency, their detailed features, and the facility which frequent recurrence affords for their collection. It is easy to explain the paucity of facts. Observation under conditions of distress is not easy. especially when minute precision is needed. A graphic presentation of visual sensations, such as that which was afforded by Dr. Airey, can only be recorded from memory, because vision is too much interfered with by the subjective disturbance at the time. A knowledge of, or at least some acquaintance with, the physiology of sensation is necessary for the full use of the opportunities which are met with, especially by personal observation. It is the hope that $I$ may excite some to careful record, that makes me choose this subject for consideration, and describe some cases which illustrate the leading features of these strange mani festations. I will, however, endeavour to avoid repeating those that I have given in the Bowman Lecture on Subjective Sensations of Sound, and in the lecture on migraine in. The Borderland of Epilepsy. I do not pro. pose to discuss their nature, although it is difficult to avoid alluding to the hypotheses that have been brought forward.

The prodromas are a functional disturbance in the sensory region, and so is the headache which succeeds. But the headache may be termed a coarse disturbance consisting of pain, often most intense, and sometimes with some secondary effects of its severity. On the other hand, it may be slight and of short duration, and it may be even absent, the prodroma oceurring alone. These premonitory symptoms, although sensory, do not include pain. They are restricted in range. The sense of sight is often involved, sometimes by loss, partial or general, sometimes by an elaborate display of its higher crude functions in light, colour and apparent motion. Other special senses do not participate, except the sense of touch. Neither smell, taste, nor hearing seems to be involved, although there is rarely a slight degree of vertigo, the precise nature of which is not clear. The involvement of cutaneous sensibility seems to be of touch, and is a frequent and 
marked manifestation. The arm is its most common seat. It may be felt in the tips of the fingers, passing along them, and going no further, or as a zone of tingling around the wrist, which slowly ascends the arm, in the course of ten to fifteen minutes. As it passes up, it leaves behind it lessened sensibility, which gradually becomes normal, and with this some degree of loss of power, apparently the result of inhibition. After reaching the shoulder, the tingling hardly ever descends the trunk to the leg, although it may in rare cases be felt in the foot, and sometimes ascends the leg. Often, after ascending the arm, or primarily, it is felt at the month, in the lips, sometimes in the gums and tongue, on one side, but in the lips it is often felt on both sides. Here it is an intense tingling, sometimes compared to pins and needles, appa. rently as slight pricking, but it is never described as pain. It seems here also to leave distinct diminution of sensibility behind it, but on this we much need further observations, on the degree and duration and character of impairment.

Still more frequently the premonition consists in interference with sight. There may be impairment of one half of the field of vision, seldom, if ever, so complete as it is from organic disease, not reaching actually the middle line. Often the loss on one side is associated with a luminous spectrum, of which a typical form is that figured by Dr. Airey in the Philosophical Transactions. This begins as a star, a little to one side of the fixing point, which enlarges, becoming first an angled sphere and then a serrated or zigzag outline which breaks below, and the elements of which it consists become progressively smaller towards the free extremity. They are often coloured, adjacent ones differing. Sometimes they are luminous without colour. Often they are double. Within the zigzag, as it expands, there is a misty luminosity, which becomes fainter internally, and hinders sight if an object is looked at. Thus the dimness of vision within the bright or coloured zigzag out. line resembles the numbness left by the tingling as it passes up the arm. After its expansion, the spectrum often ends by a whirling explosive appearance. In other cases an angled light appears near one lateral edge of the field, or both, sometimes several interlacing zigzags. Or a stellate bright object may appear on one side and remain, without expanding. In other cases a sort of luminous lacew.ork occupies the middle of the field, sometimes bounded by a brighter band. Various other spectra are seen, of which we much need precise drawings.

This sensory disturbance may be always the same in some patients, in others varying. The premonition is often unilateral in character, and the succeeding head. ache is felt on the opposite side of the head. Sometimes the premonition is felt on both sides or has no lateral significance, and then it is succeeded by a medial or bilateral headache. These constitute the common rule, but exceptions are frequent; at least, a patient may fail to recognize a one-sided seat of the headache which succeeds a unilateral prodroma. The headache may be successively bilateral, that on one side ceasing when pain dèvelops on the other side. Moreover, the premonition when unilateral may pass beyond the middle line in its course, although the headache is confined to one side.

One other: frequent symptom of the warning remains to be mentioned. When the sensory disturbance is rightsided it is often accompanied by impairment of speech of the character of aphasian Sometimes the loss is almost complete; in other cases there is only a difficulty in the use of words. But we need much to know more precise details of this loss. when it occurs.

Of the process which induces the premonition we have only hypothesis to rest on. There is clearly a disturbance of function in certain parts of the cerebral hemisphere, probably the cortex, which is the subsequent seat of the pain. The distarbance of function is apparently crude, and seems, as it were, to ripple through the centre, leaving an inhibited condition which quickly passes away.

Any hypothesis presents difficulties. That which is least difficult is the opinion that local vasomotor spasm causes anaemia in a certain region, and that subsequent dilatation of the arteries is the cause of the subsequent pain. There is often superficial pallor and coldness during both the sensory premonition and the early part of the headache, but it is not always con. spicuous, and the state of the surface is only evidence of the occurrence of vasomotor derangement, and not of its kind. A greater difficulty is constituted by the uniform character of the nervous symptoms in most cases, and the difficulty in assuming that such a nervous discharge can be explained by such a process as vasomotor spasm. But the production of an effect may be facilitated by repetition. Some time after a previous headache an attack may be brought on by over-fatigue or by error in diet, which would have no effect soon after one. This has given rise to the assumption that some toxic state is developed in the system. It is, I think, certain that the sufferers often have a pronounced gouty inheritance. But the assumption of a peculiar blood state throws no light on the immediate mechanism of the attacks, and is quite compatible with the theory of vasomotor disturbance. Certainly marked benefit is often obtained from the regular administration of nitroglycerine (with strychnine), which seems to act by steadying the vasomotor nerves and rendering them less prone to derangement. I have never had an opportunity of observing the retinal vessels during a sensory prodroma, but during the headache I have seen no abnormality in size, and the known symptoms during vasomotor spasm of the retinal artery bear no resemblance to those which precede migraine.

The time of life at which migraine begins varies much. Often the headaches are said to go back to the period of earliest memory; they may begin only when adult age is reached, or a prodroma is added to those which before occurred without one. They may commence at any later period, and often as late as fifty or after. At any adult age, the prodroma may occur alone, at least sometimes, the head ache being or becoming slight and trifling. When a visual spectrum has often preceded the headache, it is sometimes called into separate existence without succeeding headache by any flickering light before the eyes, such as a reflection from rippling water. A surgeon, who had suffered from migraine for many years, sometimes set up his customary spectrum by the reflection from the knife with which he was operating. Thus the functional disturbance is apparently the same as is produced by actual vision.

In middle or later life the occurrence of an unaccus. tomed premonition may give rise to much concern, especially if it involves the arm, lips, or speech. It may seem like a vascular lesion in the brain, especially if it occurs without succeeding headache, or if this is only slight. Even a more severe degree of pain in the opposite side of the brain may seem the effect of a presumed cerebral lesion, to the medical practitioner as well as to the patient, who is likely to consult his adviser in alarm on account of symptoms which are new in his experience. I have known this to occur, and an erroneous diagnosis to be made without any other foundation. The symptoms which alarm the patient closely correspond to the premigrainous premonition in detailed character, and this should therefore be familiar, for the correspon. dence may always be trusted. I will indicate the points on which reliance may be placed in describing some cases to illustrate the general facts I have men. tioned, as I now propose to do. Especial weight may be placed on the deliberate progress of the symptoms; such as the sensation of numbness ascending the arm, and on the definite numbness in the lips and its extension to both sides of the lips, and on the brief defect of sensation which follows it, and on the affection of vision, and the definite but transient interference with speech. This is always associated with right-sided symptoms in vision or the arm or face. The constant vanishing of the symptoms in the order in which they come on is also of great significance.

A young man of $17 \mathrm{had}$ suffered from migraine since 12, the intervals ranging from a week to several months. Coloured zigzsgs appear on the right side of the field, and he cannot see on the right side. The spectrum expands and passes to the left, on the right side. The spectrum expands and passes to the left, together with the impairment, which thus involves at last the
whole field. After it has passed away, speech is almost lost ; for half an hour he is only able to utter a single word of four or five he wants to say. When this ceases the headache begins at the back of the left eye, passing to the left side of the head, for six hours or so, it persists throughout behind the eye. There was no sickness, but great general sense of cold, and a very small pulse.

A man of 37, who had had rheumatic fever in early life, had suffered from left-sided headaches for a year, about once a month. They began sometimes soon after waking in the morning, more often at any time in the day. First there is 
numbness in the tips of the fingers of the right hand, which passes slowiy up the arm and then is felt in the right side of the cheek and mouth, but does not go to the throat. He is then unable to utter words correctly; there is not difficulty in getting the right word, but a difficulty in uttering it, apparently in articulation. Then this and the numbness cease, and headache comes on in the left frontal region, or left side of the occiput. On nitroglycerine the attacks ceased for nearly a year, and they returned less frequently and in very slight degree, but with the same premonition, and he had occasionally, during sleep, a right-sided convulsion, apparently of idiopathic epilepsy. T'here was no optic neuritis or sign of organic disease.

A member of our own profession, aged 37, whose mother also suffered from migraine, had had attacks as long as he could remember. The headaches had been preceded by visual symptoms, which occurred alone once or twice a week when he was seen, without succeeding headache. The condition begins as a luminous zigzag near the periphery on one side; it becomes larger, without colour, and inside it is a yellowish luminosity. Then it seems to him to cross to the other side, but there is no definite hemianopia. The side varies on which it appears, but the headache he had previously was always on the side opposite to that on which the spectrum began, and was accompanied by a small spot of hyperaesthesia on the occipital scalp. Formerily, when the premonition was on the right side and the headache on the left, aphasia formed part of it, and the first symptom was numbness in the hand, beginning in the hypothenar region and passing along the tips of the fingers to the thumb, which passed off in the same order, and never ascended the arm.

When a prodroma in the arm is associated with disturbance of vision the former usually occurs first. But it occurred last in one patient :

A lad of 18 suffered from headaches, which came on at intervals of six weeks or longer, sometimes six months, and then two or three headaches occurred at much shorter intervals. First there is an appearance of zigzag shaped objects before the eyes, not coloured, extending over the flelds, but intermittently, and anything at which he is looking disappears and then can be seen, again to vanish. This is repeated several times, and is followed by slight dimness of vision to one side. Then numbness occurs in one hand or the other, sometimes in the shoulder, and then it passes down the side and the leg. Sometimes it passes up to the face and involves the tongue and throat, like pins and needles, on one side only, but the side varies in different attacks. It is slow in progress, taking half an hour to go through the side, and seems to recur in waves in its course, as did the affection of sight. Sensation in the skin seems dimmed by it, but is not lost. When it is over headache comes on, severe, usually on the side of the head opposite to that on which the prodroma was felt, rarely on the same side, lasting from one to several hours, and attended by vomiting. I could not learn the character of the premonition of the same-sided headache.

In almost every case which is carefully studied the prodroma presents some special peculiarity, and a number of precise records must be collected and compared, to furnish the ground for any inference regarding their features. Momentary symptoms are usually characteristic of the warning of epilepsy, but in one case of migraine the prodroma was a bright spot of light, with a blue margin, which lasted only for a moment, but returned three or four times in the course of an hour, when severe pain came on, on one side of the head. The patient was a woman, 35 years of age, who had suffered thus for many years. Her mother also had migraine.

I have said that in bilateral or medial headaches, if there is a prodroma, it is of similar distribution. Thus a man, whose attacks of pain were severe and were situated in the middle line of the head, experienced a premonition which consisted in a concentric contraction of the field of vision, which became restricted to a small spot in the middle of the normal field. Before this he sometimes had tingling in both hands. In another case there is first general dim. ness of sight and after it has lasted a quarter of an hour, bright luminous spots appear at each outer side of the conjoined field of vision, and are seen in rapid movement. They do not extend far into the field, and one or two are larger than the others. When they disappear, headache comes on across the forehead and behind both eyes. It lasts only an hour, but he feels tired for the rest of the day.

Migraine and epilepsy occasionally coexist in the same patient, as in the second case mentioned above; but generally whicherer disease existed first presents a diminution : after the second has developed. That truis is tive ruie cannot be doubted if a large number of cases are compared. I pointed this out in the Border. land of Epilepsy, and mentioned some examples. The two affections seem, however, essentially independent. I have only once or twice met with a true connexion between them, the visual premonition of migraine becoming quickened into the aura of the epileptic tits. But such a relation is extremely rare. As a rule, the prodroma is always most deliberate, and is never attended by motor spasm, or, indeed, any motor symptoms, except the slight weakness when the numbness has just passed. Very rarely some spasm may be met with in association with the sub. sequent hradache, when it is very intense. In a clergyman of 47 , whose headaches began at 20 , the early symptoms were unusual, inasmuch as right hemianopic dimness of sight, for a quarter of an hour, was followed by right-sided headache, chiefly at a spot in the right side of the occiput. The pain also passed down the spine to both legs and caused a twitching backwards, apparently semiconvulsive. The pain reached its height in six hours. There was no change in consciousness. The motor spasm was clearly a secondary effect of the pain. It had given rise to the diagnosis of epilepsy, and also of hystero. epilepsy, to neither of which it had any relation.

In another case, a woman with very severe headaches of long duration-two days-which were attended during their height by semiconsciousness, and sometimes by spasm on the left side when the headache had reached its intensity, on one occasion the spasm made her fall out of bed. The prodroma was also long, lasting two hours, and consisted of coloured zigzags, which sometimes occurred alone.

That which is presented to the patient in the sensory premonition is a release of function, the form of which is thereby shown, and also its character. It is this which gives importance to the precise details. Most of the unilateral premonitions may be explained by the limitation of the disturbance to one hemisphere, by whatever it is excited, arterial spasm or whatever may be productive of the activity of function. But in rare cases the visual loss has the form of transverse hemianopia, loss of the upper or lower half of the combined field. In the hinder part of each hemisphere is represented the lateral half of the field, as a combination of the upper and lower quadrants. But the transverse loss implies an affection of the two upper or two lower lateral quadrants, situated in different hemispheres. This may be effected by arterial spasm involving symmetrical branches in the two hemispheres, on the supposition that arterial spasm excites the initial disturbance ; or it is conceivable that this spasm may be on one side only, and the functional inhibition induced may act secondarily on the related centre in the other hemisphere.

I have elsewhere mentioned some examples of transverse hemianopia, loss of the upper half. ${ }^{1}$ It was also described by a woman, aged 38, as a prodroma of headaches from which she had suffered for twenty years. The first symptom was loss of the lower half of the combined field, observed with each eye. Often, but not always, luminous zigzags were seen in the dim region, bright but without colour. During recent years this had been followed by other symptoms, numbness and tingling in the upper lip or in one thumb, and on one occasion a patch was felt on the back of the left foot. Sometimes it was felt on the right side of the nose and adjacent cheek, with marked diminution of sensibility. When on the thumb it may remain there or may ascend the forearm as far as the elbow. Wherever it is felt, it lasts for ten or fifteen minutes, and then the headache begins, usually at the back of the head, and comes forward.

In another patient, a woman of 41 , the hemianopia was sometimes lateral and sometimes transverse; the lateral loss did not extend to the middle line of the field, but the transverse loss reached the horizontal mid-level. If she looked at the middle of a closk dial, everything below the middle was indistinct. There was also numbness in the arm, slowly ascending and going to the lips and mouth on that side. When this was on the right side, loss of speech occurred also before the headache.

In young children sudden symptoms are sometimes seen, with headache, pyrexia, and general aspect of illness, often with vomiting. The symptoms cause a fear of acute meningitis, but after one or two days they rapidly pass away. For example, a boy, aged 9, had suffered since the age of 6 from two or three such attacks about every 
six months. Each began with frontal headache for an hour; then extreme sleepiness came on, with vomiting at intervals. The temperature rose to $101^{\circ}$ or $102^{\circ}$; the tongue was much furred but after thirty.six or fortyeight hours all the symptoms passed away. There was a strong double family history of gout. The attacks generally seemed to be excited by some indiscretion in diet. I doubt not you will remember such cases, which come chiefly under the notice of the practitioner rather than of the physician, and I should like to know whether you have not observed that they gradually acquire, as years go on, the characteristic features of true migraine.

At the other end of life the prodroma of migraine frequently occurs without, or with but slight, headache, and sometimes with more brief headache than occurs during middle life. Occasionally the loss of power seems to be the chief symptom, and in these cases the headache is generally so slight as not to attract special attention. With many patients an error in diagnosis readily occurs in a first attack of this character, although another one is less likely to be mistaken. The weakness may be considerable. In one patient the attack came on when on a journey, and for an hour he walked with great difficulty. If we assume that vasomotor spasm is the cause of the prodroma, we may regard arterial spasm in the motor region as a sufficient cause of the loss of power.

The same conclusion was reached by Dr. Langwill of Leith regarding two cases of this character in late life, in which symptoms quite like those of a grave organic lesion passed away after an hour's duration. Each patient had other similar attacks. ${ }^{2}$

Recovery may not always occur, even if the vasomotor hypothesis is correct. The arterial contraction prevents sufficient blood reaching the region of the brain to maintain its function. When the blood state favours rapid thrombosis this may occur in the vessel so as to preclude the return of the circulation, and enduring thrombosis, with all its consequences, may maintain the loss of function as permanent hemiplegia. Indeed, there is some danger of this in earlier life if any change in the blood promotes its coagulation. A woman of 29 had been sub. ject to migraine as long as she could remember, always on the right side of the head. During the fourth month of her second pregnancy an attack of her customary pain was followed by left-sided weakness, with ataxy of the hand, permanent left hemianopia, and impairment of sensibility on the left side. Pregnancy often involves an increased proneness to blood coagulation; there was no heart disease or albuminuria.

Migraine abounds in problems of interest, but $I$ have striven to confine your attention to the points on which additional and extended facts are much needed. A consideration of the cases I have mentioned will show how numerous and various are these features in every case.

If these are not at first evident to you, I shall be only too glad to have an opportanity of pointing out the details on which any case may add to our knowledge, on information as to the general character of the premonition. The task is not easy, for the prodroma is brief and ever changing, and observation needs the perception of another person; but it is worth an effort and will reward the trouble it occasions.

I may add another word regarding treatment. The benefit from nitroglycerine and strychnine, although often most conspicuous, is not invariable. Cases in which vasomotor disturbance is well marked externally sometimes derive no benefit. Why this should be the case it is not possible to say. Bromide taken regularly may then have more effect, or a small, long. continued course of salicylate. For the relief of attacks which are developing, a dose of antipyrin has often a quick influence, or Hochst's fused combination of antipyrin and caffein (migrainin), or a similar preparation made by Martindale of antipyrin fused with salicylate of caffein (migralgin). Nitroglycerine may conveniently be given in the form of the 1 per cent. solution, liq. trinitrini, but the mixture containing it must be kept acid. It may therefore be usefully united with a digestive tonic. Dyspepsia is common in some of those who suffer from migraine, and an error in diet may give rise to an attack some time after the previous one.

\footnotetext{
REFERENCEs.
1 Borderland of Epilepsy, pp. 59, 84, 97. . ${ }^{2}$ Scottish Medical and

\section{Ant Adurress}

\section{THE DIAGNOSIS OF SYPHILITIC IISEASLS \\ OF THE NERTOUS SYSTEM.}

Delivrred at the Clinical and Pathological Srction of the Birmingham Branch of the British Medicat Association, on Friday, April 30th, 1909.

By F. W. MOTT, M.D., F.R.C.P., F.R.S., PHYSICIAN TO CHARING CROSS HOSPITAL, PATHOLOGIST TO THE LONDON COUNTY ASYLUMS.

AцtнобGн syphilis is directly or indirectly responsible for a large number of organic diseases of the nervous system and it is a useful practice based upon the results of experience to treat a large number of cases of disease of the nervous system by antisyphilitic remedies, yet it is well to bear in mind that in such a widespread disease as syphilis, coincidence as well as cause is of very frequent occurrence. Therefore, in making a diagnosis we have to determine the question whether the fact that the patient has had syphilis, or even presents at the time of examination signs of active syphilitic lesions, is a coincidence or bears a direct or indirect causal relationship to the nervous symptoms for which he is consulting you. In many cases, especially in women, there may be no history of syphilitic infection and no signs on the body, and yet the character of the disease may clearly point to the possibility or even the certainty that it is of syphilitic origin; or it may happen that with: out a history or signs of syphilis a nervous affection may arise in an individual, which after antisyphilitic treatment ends by cure, arrest, or amelioration, but it does not necessarily follow that the disease was syphilitic, for we do not know what would have happened in the way of improvement if no drugs had been administered; in fact we cannot be sure whether it was an example of coincidence or cause.

A person suffering with an affection of the nervous system, whose blood gives a positive antigen reaction, is much more likely to be suffering from a syphilitic affection than one who has had syphilis but whose blood does not yield the reaction; for it is an indication that the syphilitic virus is still active, and therefore capable of producing true syphilitic lesions of the nervous system. An examination of the cerebro-spinal fluid in such a case will afford most valuable evidence not only as to whether the disease of the nervous system is post-syphilitic or not, but whether it is one of true syphilitic inflammation or of a degenerative parasyphilitic nature. Such evidence is of vital importance, for, in the case of the former, mercury, rapidly but judiciously administered, may be followed by a complete cure, whereas in the latter it will do more harm than good. I will cite a case that was recently under my care.

A woman, aged 34, was admitted to Charing Cross Hospital said to be suffering from tabes. She had shooting pains in the legs, unsteadiness in gait and station, a feeling of the soles as if walking on cork, unequal pupils which reacted sluggishly to walking on cork, unequaion, pain and cramp in the muscles of light and to accommodation, pain and cramp in the muscles of the legs, absent knee-jerks, patches of anaesthesia on the legs,
and a belt of thoracic anaesthesia with girdle sensation. After inquiring into the history and finding that she had suffered with headache and squint, that the knee-jerks which were absent on admission had returned a few days later, I came to the conclusion that this woman, with a probable duration of infection of not more than four years, was suffering really from pseudo-tabes the result of syphilitic meningitis, especially as pseudo-tabes, the result of syphittic mening in the neck, and I then obtained Kernig's sign. Lumbar puncture was performed, and 390 lymphocytes per c.mm. were found-8n enormous number for tabes dorsalis. This large number of lymphocytes could only be accounted for by a widespread active gummatous change in the meninges. She was placed on mercurial inunction. The symptoms began to clear up rapidly, and a fortnight later thelymphocytes had fallen to 70 per c.mm. and the fluid this time was tested by the Wassermann method, and the fluid this time was tested by the Wassermann method, and found to give a negative reaction for both antigen and anti-
body. Unfortunately, blood was not tested on this or future body. Unfortunately, blood was not tested on this or future
occasions. A fortnight later the cerebro-spinal fluid was examined and only 20 lymphocytes per c.mm. found, the patient being almost well. A fortnight later still there were no prmphes reaction. The patient was quite well; the pains, anaesthesia, and unsteadiness had entirely disappeared. 